\title{
Corela
}

Cognition, représentation, langage

HS-19 | 2016

Le point de vue pris au mot

\section{Le pathos mis à contribution dans les discours à grande circulation}

Danielle Forget

\section{(2) OpenEdition}

Journals

Édition électronique

URL : http://journals.openedition.org/corela/4434

DOI : $10.4000 /$ corela.4434

ISSN : $1638-573 \mathrm{X}$

Éditeur

Cercle linguistique du Centre et de l'Ouest - CerLICO

Référence électronique

Danielle Forget, «Le pathos mis à contribution dans les discours à grande circulation », Corela [En ligne], HS-19 | 2016, mis en ligne le 08 juin 2016, consulté le 01 mai 2019. URL : http:// journals.openedition.org/corela/4434; DOI : 10.4000/corela.4434

Ce document a été généré automatiquement le 1 mai 2019.

\section{(c) (i) (2)(2)}

Corela - cognition, représentation, langage est mis à disposition selon les termes de la licence Creative Commons Attribution - Pas d'Utilisation Commerciale - Partage dans les Mêmes Conditions 4.0 International. 


\title{
Le pathos mis à contribution dans les discours à grande circulation
}

\author{
Danielle Forget
}

\section{Introduction}

1 Dans la présente étude, je m'intéresserai à certaines configurations discursives dont l'agencement linguistique et argumentatif est fortement récurrent dans les discours à grande circulation, tel le discours politique. Elles mettent au premier plan les affects, ce processus favorisant, me semble-t-il, une valeur persuasive prépondérante.

2 Sans travailler directement les concepts qui fondent la recherche de Pierre-Yves Raccah, mon propos s'élabore dans leur prolongement. Nos collaborations passées et nos discussions actuelles cherchent, justement, à mieux établir ce point de jonction. L'identification des configurations discursives vers laquelle tend mon analyse fournit une matière dont se nourrit le sens des énoncés. La sémantique linguistique que pratique Pierre-Yves Raccah a pour tâche la reconstruction formelle de l'activité de compréhension, d'où son intérêt pour les phrases de la langue et leurs instructions sur le sens des énoncés. Comme il le formule «[...] la signification de la phrase est l'ensemble des contraintes que la langue impose sur la construction du sens [...] » (Raccah 2005 : 209).

Dans cette étude, à l'aide d'un cadre théorique appartenant à l'analyse du discours, j'amorce une réflexion qui se situe à la croisée de la sémantique, de la pragmatique et de la rhétorique, dans cet espace discursif où les énoncés accusent la présence de schémas culturels à forte récurrence. Ces schémas sont en cela reconnaissables lors de la circulation des discours sur la scène publique, en même temps qu'ils reformulent et renouvellent une prise de position idéologique à l'intérieur des nouveaux paramètres textuels qui se présentent. En effet, il faut considérer que ces discours prennent place dans une tension entre le même et le différent qui assure un caractère à la fois consensuel et dynamique à la prise de parole. 


\section{Les émotions sous la loupe} émotions afin de mieux cerner le lien entre les composantes cognitives, sémantiques et pragmatiques du discours. Le locuteur livre volontiers ses émotions face à un événement qu'il cherche à décrire, à exposer : le contenu propositionnel passe par la composante émotionnelle, cette dernière étant fortement ancrée dans un lieu, un temps, tout en étant en rapport avec des éléments interactionnels ${ }^{1}$. expression, cependant. Cosnier (2006) insiste sur la transmission, qu'elle soit inconsciente ou non, autrement dit sur un partage entre locuteurs et destinataires. Cela signifie-t-il que l'émotion, dans l'échange interactionnel, se déposerait d'un individu à l'autre comme en des vases communicants, exactement la même qu'à la source ? Évidemment pas, et c'est justement le rôle des analyses sémantiques et pragmatiques d'en entrevoir les mécanismes d'interprétation et de réception.

Ces études remontent volontiers à l'expérience qui servirait d'élément déclencheur à ces émotions ; les événements en sont bien souvent à la source et entraînent des effets qui vont au-delà de l'épisode immédiat, révélant ainsi la complexité des attitudes impliquées. Ainsi, l'analyse des mécanismes suscitant l'état émotionnel permettrait de dégager des associations stéréotypées à partir d'éléments déclencheurs, et de reconstruire les types d'émotion. On pourra mettre les émotions à contribution dans l'analyse des interactions conversationnelles, plus spécifiquement dans leur rapport à la prosodie. Temps et rythme de la parole constituent alors les points d'intérêt sur lesquels se porte l'analyse des émotions (Auchlin 2003).

7 De plus, Plantin souligne pertinemment qu'il ne s'agit pas là d'un phénomène exclusivement individuel :

Bien que, dans la plupart des exemples allégués, on considère que l'événement inducteur affecte un individu privé, coupé du monde pragmatique et du groupe interactionnel [...], rien dans ce modèle n'oblige à considérer que l'émotion est fondamentalement un événement privé; le même événement peut affecter simultanément tout un groupe. (Plantin $2003: 102$ )

8 La cohésion du groupe quant aux valeurs et aux intérêts défendus induirait en quelque sorte une communauté émotionnelle : « il partage ou co-élabore des états émotionnels du même type, l'émotion de chacun se renforce de celle des autres » (Plantin 2003 : 102).

Ces études proposant une approche de l'émotion ont réussi, ces dernières années, à faire ressortir une facette négligée de la construction du sens. Mon propos, dans ce travail, en respecte l'orientation générale. Je serai amenée à utiliser la notion d'empathie, qui consiste en un partage d'émotion dans les relations interactionnelles, en rapport avec un thème ou un événement. Toutefois, mon objectif consiste à jeter un éclairage sur ce qu'il convient d'appeler la charge pathétique en contexte d'argumentation. En outre, cette charge pathétique se trouve liée, comme nous le verrons, à un type de configurations discursives, et celles utilisées au sein du discours politique serviront d'illustrations ${ }^{2}$. Comment ces configurations se chargent-elles de pathétique et, en conséquence, comment exercent-elles leur rôle persuasif? 


\section{Pathos et persuasion rhétorique}

10 On ne saurait bien saisir comment les affects peuvent s'inscrire dans le discours sans passer par le rôle dévolu au pathos en rhétorique. Les affects, conformément à la vision qu'en a Kant, sont ces manifestations du sensible et les traces qu'ils laissent chez l'individu qui les expérimente. Considérons les passions comme, parmi les affects, ceux affublés de plus grande intensité et qui participent d'une intersubjectivité mise en rapport de force dans le discours. Or, le pathos dispute sa fonction dans ses rapports avec l'ethos, mais surtout avec le logos auquel on l'a souvent opposé.

11 On ne peut passer sous silence le fossé qui s'est creusé entre la raison et la passion; il n'est qu'un des témoignages du parcours différentiel subi par logos, ethos et pathos dans le curriculum académique, ces trois types de preuves reconnus par la rhétorique ancienne. La valorisation du logos trouve sa quintessence dans la logique et ses dérivés en termes de raisonnement. L'appareil formel développé pour rendre compte de l'argumentation, telle que travaillée par le logos pourrait-on dire, atteste la complexité de son intervention dans le raisonnement. Structures transposables en langage codé, schémas argumentatifs repérables, déduction de conclusions à partir de relations clairement établies. N'oublions pas qu'à l'époque de la rhétorique antique, la rigueur fut l'une des préoccupations majeures face aux sophistes, dont on disait qu'ils subvertissaient la démarche logique. Les procédures explicites et formelles outilleront l'approche centrée sur la réfutation, afin de démonter les paralogismes. Cette préoccupation est à l'origine de l'enseignement d'Aristote : dénoncer les mécanismes, identifier les structures défaillantes de leur mise en discours.

12 Attardons-nous au pathos. Dans l'optique de la rhétorique, le pathos est cette charge d'émotion provoquée chez autrui avec pour but de persuader. Une telle visée en fait un facteur qui ne se transpose pas exactement dans les fonctions de Jakobson malgré leur pertinence. Toutes les fonctions sont diversement activées, mais particulièrement l'expressive, la conative, et la poétique.

13 Rappelons que les fonctions proposées par Jakobson (1963: 52 et suiv.) sont fort générales et attachées au langage qui les distribue en plus ou moins grande importance dans les énoncés selon les situations. La fonction expressive est celle par laquelle le locuteur livre son intériorité par l'expression de ses sentiments, de ses émotions. Lorsqu'un énoncé est marqué de la fonction expressive, c'est que le locuteur parle en son nom propre, le « je », pour exprimer ses sentiments, ses émotions. La fonction conative est celle qui est sollicitée expressément dans l'adresse au destinataire (ex. : par un conseil au moyen de l'impératif). Il ne faudrait pas croire la fonction poétique réservée à la poésie ; même si cette dernière en exploite de façon privilégiée les artifices, la fonction poétique s'exerce lorsque viennent les transferts de sens, lorsque l'arrangement du texte est lui-même porteur de sens, complice du message à transmettre.

14 Le pathos se présente à travers des stratégies de communication par lesquelles le locuteur mise prioritairement, pour la persuasion, sur ses propres affects et leur transmission. Les affects apparaissent dans l'énonciation avec une charge telle (au sens d'intensité) qu'elle est susceptible d'influencer le destinataire, contribuant en conséquence à la persuasion. Bien sûr, il arrive que la transformation des opinions d'autrui n'est pas explicite: dans certaines situations, on peut échanger du pathos par compassion, par exemple, sans que les objectifs persuasifs soient au premier plan. Malgré tout, la persuasion ou du moins, la 
transformation des rapports entre énonciateurs s'en trouve tout de même affectée en bout de ligne. Ainsi, pathos et interaction sont intimement liés.

Il reste qu'en présence des locuteurs concernés, autrement dit, en situation réelle d'interaction, on peut avoir une meilleure appréciation de l'effet pathétique qu'à la simple lecture d'un texte écrit. À l'oral, en effet, la voix, le geste, la mimique, le mouvement $\mathrm{du}$ corps sont symptomatiques de telle ou telle passion et fonctionnent conjointement au discours en situation intersubjective. Mais dans la présente étude, mon propos s'attache à des observables textuels, comme manifestations exemplaires des stratégies persuasives qui, mutatis mutandis, pourraient survenir tant à l'oral qu'à l'écrit, et dans ce cas, s'intégrer à des discours sociaux variés. Je me suis donné l'objectif d'étudier comment la dimension pathétique se manifeste dans la parole publique, le discours politique recoupant le discours médiatique dans la mesure où ce dernier rapporte des interventions à teneur politique ou prend en charge, par la voie des chroniqueurs une parole avec enjeux politiques déclarés. Il va sans dire qu'une étude plus ample s'attacherait également à l'aspect audiovisuel à travers ces véhicules de l'image, du texte, de l'arrière-plan sonore et musical qui sont à l'œuvre dans la composition du pathos . Si je les écarte ici, c'est pour condenser le propos et non faute de reconnaître leur contribution au pathétique. D'ailleurs, lorsqu'il s'agit de textes, autrement dit de discours relatés, narrés, transcrits, comme ceux qui nous intéressent prioritairement, la tâche n'est pas moins ardue, puisqu'elle se prive de ces indices flagrants de la passion pris à même la présence en face à face des énonciateurs. Mais justement pour cette raison, déceler le pathétique dans l'écrit exige d'en venir à un niveau complexe de manifestations et il vaut la peine de s'y consacrer.

\section{Un phénomène intersubjectif}

Le pathos s'inscrit dans l'énoncé et participe également à l'argumentation. À propos de son repérage dans le discours, Angenot (2008: 258) soutient que les marques du pathos « ne forment pas une catégorie isolable, elles ne sont pas séparables de schémas cognitifs et des raisonnements, qui ont toujours, hors le plus pur esprit de géométrie, une "dimension" affective ». Que faut-il conclure de cette absence de marques linguistiques distinctives de la dimension affective? Cela empêcherait-il d'en comprendre les mécanismes de fonctionnement et leur rôle persuasif? Nous soutenons que le pathos se manifeste de diverses façons dans l'énoncé - que ce soit par les modalisations, les exclamations, etc. - qui sont l'indice d'une subjectivité énonciative.

À partir de ces empreintes, il est possible de faire des hypothèses sur nos représentations, c'est-à-dire sur le perceptif, l'intuitif, le « ressenti », le conceptualisé, qui se livrent en images du réel. Le pathos sera ainsi de l'ordre du "supposé » - basé sur des données conventionnelles que nous livre l'énoncé - et non de l'ordre d'un "réel» absolu. Cherchons à cerner la fonction heuristique du champ pathétique dans le discours et la construction du sens, de manière à faire ressortir les conditions d'opérationnalité d'une telle notion. À quelle condition ce concept peut-il, dans une variété de contextes, fournir une interprétation, la plus complète et juste possible, des enjeux ?

18 Je choisis d'aborder le pathos en tant que construction discursive ${ }^{3}$; par conséquent, ce sont les discours où il émerge qui permettront de mieux en connaître les mécanismes de fonctionnement, les effets, ainsi que le rôle qui lui revient en persuasion. Il ne faudrait pas en conclure que le sens varie d'une occurrence à l'autre. Un fond sémantique général 
en organise les contours et il transparait dans les propos que nous tenons sur différents sujets. Le contexte linguistique se charge de fournir les précisions subséquentes.

La philosophie kantienne nous éclaire sur cette distinction. Pour Kant, le monde est celui livré par les perceptions. Rien à voir avec une vérité fixe, un déjà-là avant la connaissance que j'en prends, ce que l'on appellerait l'essence des choses ${ }^{4}$. Le monde passe par le filtre de l'interprétation; il est le produit de ce regard que je jette sur lui. Voilà qui nous amène à la subjectivité 5 . Une vision du monde ainsi livrée nous en apprend tout autant sur l'objet que sur le sujet. En transposant sur le plan du discours, nous dirons que non seulement le locuteur / sujet s'exprime par une énonciation, mais qu'à travers l'énoncé produit, sa subjectivité apparaît. Pour reprendre la thèse de Ducrot (1983), la subjectivité est inscrite dans la forme même de la constitution du message (voir aussi Raccah 2005 ; Simonffy 2005). Elle ne se rajoute pas de l'extérieur comme l'ajout d'une coloration particulière sur une toile blanche. Le choix d'éléments linguistiques, qu'ils soient syntaxiques, lexicaux, sémantiques, est responsable de cette "présence subjective", à laquelle peuvent se joindre des tournures stylistiques et rhétoriques qui les renforcent.

Sur la scène publique, le discours politique est souvent émaillé de traces de l'affectivité. L'orateur, qu'il soit politicien, chroniqueur journalistique, ou simple citoyen s'emploie à louanger, à dénoncer, à accuser, à rallier, peu importe la dénomination des actions explicites ou non, elles seront associées à des affects, que ce soit l'admiration, la colère, la honte, la haine, etc. ou même à une conjonction d'états d'âme. Il importe moins de poser une étiquette sur une passion en particulier que de prendre en compte la charge pathétique, donc en quoi le pathos est mobilisé dans l'entreprise persuasive. Au-delà des classements et des nomenclatures (dont les limites sémantiques et les connotations ont évolué au cours des siècles) des passions, il convient de s'attacher au repérage du pathos en énoncés - par le biais de propriétés qui restent à définir.

Citons, pour exemple :

(1) On en a vu des projets qui sont tombés à l'eau parce que ce sont les 'bloqueux' qui ont gagné. Peut-être faudrait-il qu'il y ait une victoire pour la marche en avant, pour le progrès, pour le développement du Québec, pour s'assurer que l'État du Québec puisse continuer d'assumer ses responsabilités, que nos universités auront des ressources pour se financer, qu'on pourra réparer les ponts, pour ceux qui risquent de tomber, qu'on pourra se doter des infrastructures qui nous font gravement défaut actuellement. Il y a tellement de besoins au Québec. Pendant ce temps-là, on parle, on discute, on s'accuse, on s'impute des motifs, alors que c'est en avant qu'il faut aller tous ensemble.

(Tiré de l'article «Le gaz de schiste en héritage », Alexandre Shields, Le Devoir, 24 octobre 2012, B1)

Cet extrait provient d'un discours de Lucien Bouchard, président de l'Association pétrolière et gazière du Québec et ex-premier ministre du Québec (de 1996-2001). Bouchard est un ardent défenseur du gaz de schiste dont l'exploitation fait l'objet de discussions acharnées au Québec. Mais, même sans rien connaître du contexte, on aura pu détacher un certain nombre d'éléments porteurs d'affectivité, éléments qui sont fréquemment répertoriés en rhétorique (la question, l'énumération, etc.) ou qui s'intègrent à des catégories linguistiques porteuses d'intensité ("tellement", " gravement »).

Toutefois une identification des marques, une à une, utilisées à des fins rhétoriques ne suffit pas pour comprendre les mécanismes du pathos à l'œuvre. Bouchard fait valoir ses propositions conclusives en cette portion de discours qui, d'une part comporte des 
marques de la subjectivité énonciative et d'autre part, interpelle le destinataire. En témoignent la suggestion par le biais d'une question rhétorique en "Peut-être faudraitil... ", la tournure exclamative et l'injonction sous couvert d'une mise en relief à la toute fin « c'est en avant qu'il faut tous aller ensemble ». Ce sont là des indices qui attestent des fonctions expressive et conative constitutives du pathos, de même que l'intersubjectivité convoquée.

Nous poursuivrons dans cette voie en tentant de dégager un modèle qui permette d'articuler ces procédés sur les mécanismes de persuasion, afin de comprendre à quel titre le pathos s'y trouve sollicité.

\section{La force associée à la dimension pathétique}

Afin d'être à même de saisir comment s'opère le lien entre pathos et transmissibilité, et, conséquemment, la pertinence de l'empathie, il importe de prendre en compte notre fond «relativement spontané » de conceptualisation des passions. En tout premier lieu, la façon que nous avons de parler des passions elles-mêmes, quelles qu'elles soient, devrait retenir notre attention. Des relents de tradition philosophique se sont maintenus dans le langage courant pour faire de la passion une résistance à la raison, comme si elle possédait ses propres incitations, souvent impératives. Cette idée de contrainte est à retenir, car elle exprime au mieux et pour la majorité d'entre elles, l'ancrage dans l'idée de force, glosée diversement pour dire qu'elle entraîne l'homme sur une voie qu'il assume ou qui le prend au dépourvu, qu'elle peut lui permettre de déplacer des montagnes afin de combler ses désirs, la force des pulsions plus puissantes que le reste, force d'attraction ou de répulsion.

Rappelons que, dans le domaine émotionnel des passions, mais aussi des notions abstraites telles l'identité, la nation, etc., la conception que nous en avons (leur aspect cognitif) mérite d'être prise en considération: l'image qui s'imprègne - ou leur représentation - devient partie intégrante du sens. Comme pour toute réalité linguistique, l'usage laisse des traces de sa signification. Ainsi, la « force » est manifeste lorsqu'on parle des passions. On puisera dans des expressions comme «brûler des feux de la passion » et leurs variantes : «brûler d'envie, d'impatience », « les feux de l'amour ». On aura reconnu que le feu est souvent cet élément de la nature qui incarne le plus la force, avec cette propriété qu'elle se propage puissamment et insidieusement. Des expressions idiomatiques peuvent aussi être sollicitées : " être sur des charbons ardents ».

L'étymologie fournit un témoignage convergent. Si elle archive l'origine de la forme et du sens, il ne faut pas perdre de vue que la forme retenue par les dictionnaires n'est qu'une sélection à travers un ou plusieurs usages. Qui, de nos jours, dit pathos ou pathétique renvoie à l'expression des sentiments, la manifestation d'une affectivité et même d'un état d'âme. L'étymologie de pathos n'est pas anodine: en grec, le mot signifie maladie, affection. Quant à pathétique, l'adjectif est attesté à la fin du XVI ${ }^{e}$ siècle et il évoque ce qui est relatif aux passions. Plus tard, il prendra un sens négatif lié à l'excès, au trop-plein d'émotion exprimé dans une situation donnée. Ce «trop-plein » réitère la croyance dans le défaut de tempérance pour qui s'adonne aux passions: elles dicteraient un comportement par l'excès. Nul doute que l'idée de force est bien présente.

Plusieurs études appartenant au domaine du cognitif sont compatibles avec cette vision du phénomène, notamment celle que propose Lakoff sur la colère (1987: 390 et suiv.). 
Lakoff et d'autres chercheurs associés décèlent des impératifs de sens dans notre conceptualisation par le fait qu'elle se base sur la formation de gestalts impliquant notre rapport au monde, ce qu'ils appellent le bodily expérience, l'expérience sensible. Cet aspect interfère parfois avec une conception rationnelle des événements ou des phénomènes. Concernant la rotation du soleil dans le ciel, ils remarquent que, malgré nos connaissances scientifiques modifiées depuis l'intervention de Galilée qui a bien établi que la terre tournait autour du soleil et non l'inverse, nous maintenons une vision autre qui est en correspondance directe avec des "illusions perceptives »: en témoignent les expressions "le soleil se lève à l'est ", «le soleil se couche à l'ouest ». Ainsi, ce que l'on «voit » est transcrit dans le discours et paraît avoir préséance dans l'usage courant sur d'autres énoncés plus en accord avec des vérités que nous sommes pourtant prêts à soutenir par ailleurs. Une telle vision dite imaginative - parce qu'elle procède par images mentales - marquerait ainsi plusieurs domaines de sens.

Voilà une projection dite métaphorique, au sens cognitif du terme, c'est-à-dire qu'elle fait appel à l'image mentale façonnée dans la compréhension de l'objet en question. L'appellation prend un sens plus large et plus fondamental qu'une simple figure stylistique. Rappelons qu'en études littéraires, la métaphore implique aussi un transfert d'un domaine à un autre, mais s'exprime ponctuellement, dans l'opportunité du texte qui s'élabore. Comparée à la métaphore littéraire, la métaphore cognitive s'appuie sur notre compréhension du monde, non pas dans l'absolu, mais culturellement parlant, puisqu'elle est liée à l'usage qu'en fait un groupe, une collectivité. Par conséquent, elle n'a aucune prétention à l'originalité, à l'univocité, puisqu'elle peut être partagée et apparaître en différents points du globe. Ce type de métaphore ressemble davantage à un modèle cognitif de nature anthropologique, d'où sa portée très large. Les facteurs en jeu me permettront d'esquisser des axes théoriques d'interprétation des passions, à cette différence près que le cadre théorique dont je me sers est non seulement cognitif mais discursif, conformément à mon hypothèse de départ. D'où l'importance de rattacher notre façon de concevoir les passions avec les moyens pour les exprimer, y compris ceux pour les susciter, les contrer, les exploiter à des fins de persuasion.

Les affects en général sont vus, dans un premier temps, comme un champ abstrait du ressenti qui s'appuiera sur le domaine concret du monde physique pour en faciliter la conceptualisation. D'où les expressions consacrées sur les passions qui en rendent compte : on les appelle "mouvements de l'âme » ou «transports ", autant de renvois à l'idée de force qui émerge dans notre rapport au monde. Derrière l'idée de déplacement, il y a la force qui s'exerce, et c'est là un élément primordial de notre conceptualisation de passions.

31 Je propose de faire de la force l'élément de sens intrinsèque et primordial de la notion de passion, dont le sémantisme se complète par une projection de l'intériorité (la subjectivité, le "ressenti») vers l'extérieur (expressivité, interaction avec autrui dans l'échange discursif). Issue du monde physique, la force supposera des axes de sens connexes comme l'intensité (plus ou moins importante), la durée (plus ou moins longue) en prolongement du temps, l'étendue, le déplacement dans l'espace (en fonction du poids et de la vitesse) réglant le rythme et la portée. Cette conception des passions rejoint en plusieurs points celle élaborée par Fontanille (1998) dans le cadre d'une sémiotique où les passions sont reconnues comme objets de connaissance. Il y voit un dynamisme que devrait prendre en compte une "sémantique des tensions et des degrés». Cependant, la cohérence interne du système n'empêche pas la difficulté d'application des paramètres 
proposés, de même qu'une certaine clôture de l'ensemble sur lui-même ${ }^{6}$. Par ailleurs, l'auteur insiste avec raison sur le dynamisme inhérent aux émotions, aux passions. Je ferai davantage appel au schéma de la force de Talmy (1985) pour en rendre compte : il s'agit d'une conceptualisation de propriétés renvoyant à notre expérience (gestalt) de la force, plus qu'une correspondance avec les propriétés de l'objet lui-même dans la réalité. Nous avons tous expérimenté ce qu'est le poids, l'accélération, la résistance, etc. Cette appréhension cognitive sied bien aux affects. Leur sémantisme peut être interprété, dans ses grandes lignes, à travers cette représentation imaginative du dynamisme que chacun des discours ${ }^{7}$ actualisera par la parole ou l'écrit.

Pour revenir à notre hypothèse, les affects seront conceptualisés et mis en discours comme des forces, faisant appel à celles intervenant dans le monde physique. Cette métaphorisation, exerçant le passage d'un domaine à l'autre dans la compréhension, peut être illustrée à l'aide du tableau général suivant :

Éléments de conceptualisation des passions

\begin{tabular}{|l|l|l|}
\hline Domaine des affects & > par transfert vers > & domaine physique \\
\hline amour, colère, etc. & & force \\
\hline
\end{tabular}

Sans effort, ni intention, nous conceptualisons les passions par imposition d'une image sur une autre, ou interprétation métaphorique. Est-il donc si surprenant que le corps soit $\mathrm{vu}$ comme le siège des passions? Est-il donc étonnant que notre disposition psychologique impliquée dans la jalousie ou la compassion, par exemple, fasse appel à notre rapport au monde, une expérience du corps sensible? Que ce soit dans le domaine physique de la force ou dans le domaine psychologique, c'est le corps qui établit ce lien : il est le lieu de la sensibilité8. La force repose évidemment sur le désir, cette composante différente de l'instinct chez l'animal, soutient Vincent (1986: 162), par les fonctions cognitives impliquées : «Le désir serait donc défini par le but à atteindre et justifié par la récompense, profit ou plaisir, obtenue. Finalement, le désir se mesurerait à l'intensité de l'acte qui le sanctionne ». Il sert de motivation vers une satisfaction appréhendée.

Ainsi, les tiraillements de la passion, les obstacles qui se présentent au fur et à mesure de la situation en cours, les élans de celui qui en est inspiré et même parfois dominé sont vécus, dirons-nous, comme autant de forces. Elles se manifestent en pression, en mouvement, en résistance et obstacles à surmonter, en intensité. Cette vision est compatible avec nos manières de discourir sur la passion. C'est ce lien entre le monde physique et le domaine affectif via le corps qu'exploitera abondamment notre façon de parler des passions, que ce soit par une description générale, ou particulière de leurs "symptômes ", des effets qu'elles ont sur le comportement, de leurs cibles, etc.

Considérons le domaine plus vaste de la perception, des émotions, qui s'allie aux affects, aux sentiments et émerge en plusieurs lexèmes ou expressions. L'individu a beau ressentir, être le siège des sensations, encore faut-il qu'il puisse les exprimer et en décrire les effets. Il le fait au moyen de mots comme les suivants, employés figurativement pour parler des sensations, des sentiments, empruntent abondamment au monde physique: saisi, transpercé, glacé, brûler, chaud, froid, tiède, perçant, coupé, attirant, vibrant; commotion, bouleversement, secousse, choc, ébranlement, trouble, etc. Toutes catégories grammaticales confondues, ces mots sont issus de phénomènes physiques qui ont subi une modification 
figurative dans laquelle le sens s'est figé. Dans le même ordre d'idées, se trouvent ces expressions, accompagnées de leur sens qui montre la correspondance entre le monde physique et celui des affects : adopter une position ferme = être sans concession; un regard perçant $=$ qui cherche la vérité, manifeste une opposition ; avoir de l'attirance $=$ favorise le rapprochement; un spectacle repoussant = qui incite à la distance, etc.

Mieux encore. Les mots servent souvent à décrire la sensation au moyen de l'effet qu'elle produit sur le sujet, ce qui nous rapproche encore davantage du rapport au monde et de ses traces dans la conceptualisation du sensible. L'affect devient ainsi nommé ou associé à partir de ses symptômes corporels et physiques : une voix glacée = manifestation de la peur, d'une distance $;$ les mains moites = nervosité, peur ; le souffle coupé $=$ surprise $;$ rougir = honte, aveu, colère ; claquer des dents = peur ; être saisi = étonnement ; être bouleversé, remué = sous le coup de l'émotion ; des gestes précipités = la hâte, la nervosité d'une personne.... etc.

L'expérience que nous avons d'une sensation, et ainsi l'effet qu'elle produit sur nous, sert à parler de cette entité floue, intériorisée au point de paraitre personnelle. En outre, une telle transposition par le plan physique est un processus langagier fort courant, qui aide à la conceptualisation. C'est le cas des entités abstraites mais aussi du sensible qui seront exprimés et partagés plus aisément par recours au concret, puisque ce dernier aide à stabiliser le sens et à façonner le "sens commun ». Ainsi des effets qui sont de nature physique seront plus à même de signifier la sensation par ce détour vers le concret.

Revenons au champ pathétique tel qu'il se dessine dans le discours politique et qui agit dans les configurations que je porte à votre attention. L'idée de force que je propose comme essentielle au domaine pathétique se trouve développée non pas uniquement dans un choix lexical mais transphrastique. Il s'agit de configurations discursives qui produisent métaphoriquement l'idée de force par un ensemble variable de procédés d'expression.

\section{Le champ pathétique et la transmissibilité}

Poussons plus loin l'incidence de cette dimension essentielle qu'est la force. En prenant partie de la métaphorisation des domaines, celle qui permet de transposer du concret sur de l'abstrait.

Toutefois, on n'en a pas encore terminé avec le "portrait » du champ pathétique et de son émergence discursive. Il met ses ressources à leur service. C'est ainsi qu'outre le choix des lexèmes ( $c f$. plus haut), l'agencement des énoncés, les propriétés énonciatives et la composition stylistique deviennent des éléments déclencheurs. Appuyée d'autres signes convergents, une énumération, par exemple, deviendra l'indice d'insistance, de renforcement. Dans l'extrait tiré du discours de Bouchard, la série de verbes (« on parle, on discute, on s'accuse, on s'impute des motifs») tient lieu de contrepoint à la thèse soutenue : il dénonce ce qui s'oppose à l'action commune préconisée.

41 Ces configurations prolongent l'argumentaire pour implanter jusque dans le mode de l'expression l'idée de force.

42 Procédés d'expression convertis en force

- la répétition $=$ indice d'insistance

- l'exagération = indice d'intensité

- l'énumération = indices de renforcement, du poids des arguments à propos d'une cause 


\section{à défendre}

- le développement (en termes de longueur de texte) = indice d'étendue, d'expansion

- des actes de parole susceptibles de menacer la face = indices de tension

Il y a fort à parier que la répétition soit associée à l'insistance chez nombre de locuteurs dû au poids de l'apprentissage scolaire: les figures ont reçu, en effet, des formulations stéréotypées qui refont surface en ce contexte. Plusieurs de ces transferts opèrent ainsi sur la base de stéréotypes culturels activés par notre formation académique. Mais il y a surtout notre expérience de l'interaction verbale qui entre en jeu et nous porte à associer des « attitudes » à ces procédés (Forget 2009 : 74).

Bref, les facteurs favorisant l'association peuvent être divers. Le fait demeure: les procédés d'expression en question sont projetés dans le champ pathétique et prennent une valeur en liaison directe avec la transmission de subjectivité. La force d'affect que semble tenir le locuteur est susceptible d'être « vécue » à son tour par le destinataire.

L'empathie viendrait de cette « re-création » de la force au moyen du transfert concret abstrait dont la conjonction des procédés d'expression sont porteurs.

Par ailleurs, il importe d'établir certaines distinctions et de se distancier d'emplois qui ne concernent pas directement notre propos. En général, les «mentions» d'affectivité seront mises de côté au profit des « usages ». Ainsi, dans un énoncé comme :

(2) En état de choc, je n'arrivais pas à exprimer ma colère.

la colère, qui est un affect particulier, est thématisée, autrement dit elle fait l'objet de la prise de parole ; c'est ce dont on parle. Un tel énoncé n'exprime pas à proprement parler la colère, même s'il en est question. En effet, nous privilégions les énonciations manifestant de l'affectivité au point où cette dernière semble suscitée dans le moment même de la prise de parole.

Dans les configurations que nous étudions, la situation d'éclosion de l'affect n'est pas relatée, comme survenue avant le discours, mais plutôt prend place dans ce dernier, comme s'il s'y trouvait des éléments déclencheurs. Dans ces occasions, le présent de la narration a tous les pouvoirs. Ainsi, le locuteur peut très bien raconter une colère qu'il a éprouvée dans le passé alors qu'un ivrogne a provoqué un accident de voiture : son récit " réanime » la colère chez lui. D'où l'enclenchement de la forme du discours qui acquiert des attributs de la force tout en tirant parti d'un effet énonciatif d'immédiateté. Le lecteur semble alors invité à réagir en symbiose avec le locuteur / auteur lui-même. Les mécanismes discursifs seront sollicités pour accentuer ce rapport de correspondance entre la passion et les propriétés dynamiques du monde physique aux prises avec l'intensité du moment. Le combiné métaphorique (univers de la passion / monde physique) est converti dans le discours qui, par ses propres ressources, achève d'établir la correspondance. Cela ne va pas sans rappeler le rôle dévolu aux interjections, ces mots dont on dit qu'ils sont « comme arrachés par la situation ». Lorsqu'un « oh! » intervient, l'on se sent pris dans la surprise provoquée et exprimée. Cet accompagnement du moment est bien différent de la référence à la surprise contenue dans (3)

(3) Je suis surpris chaque fois que retentit la sonnerie

même s'il est question de surprise dans les deux cas. Avec l'interjection, l'affect est constitué, semble-t-il, dans le moment même du discours.

50 Cette métaphorisation convertissant les procédés d'expression en force fait que le discours acquiert une nouvelle dimension d'actio, pourrait-on dire en reprenant le terme dévolu à l'une des étapes de la démarche rhétorique, et qui a trait à la mise en discours : 
celle où l'orateur, en contact avec son auditoire, est appelé finalement à livrer «live» son discours à partir de techniques mettant en valeur sa présence. Il est fort à propos dans cet usage « actualisé ». La passion n'est pas alors un objet de référence ; elle est «jouée » en quelque sorte dans le moment présent de l'énonciation (la prise de parole). Cette propriété, que nous appelons actualisation, tend à neutraliser la différence entre la référence, c'est-à-dire le narré (ou la mimesis, s'il s'agit d'un récit), et la narration, comme si le sujet expérimentait hic et nunc l'affect en question, ce qui favorise l'empathie. La force est perçue grâce à la valeur temporelle du discours se faisant, bref une valeur performative en quelque sorte, ce qui fait que la dimension affective paraît se développer dans l'instant même où prend place le discours. Nul doute qu'une telle actualisation est de nature à favoriser l'empathie car le destinataire est sollicité également à ressentir cette force émergente dans le moment même du discours. La performativité qui se dégage de ce type de configuration est garante d'authenticité, un argument facilitant l'adhésion, comme on le sait.

51 Par ailleurs, il faut se garder d'associer exclusivement les affects avec l'expression d'une intériorité. Il est bien évident que les sentiments sont vécus par l'individu; comme les perceptions et les émotions avec lesquelles ils sont proches parents, les sentiments trouvent leur siège dans l'individuation. Ils naissent, se livrent ou se refoulent, atteignent divers degrés, peuvent s'évanouir, se transformer : ils sont intimement liés au vécu des uns et des autres. Toutefois, les affects, du fait qu'ils sont mis en discours, sont façonnés par les ressources de ce dernier parmi lesquelles figure l'empathie, qui passe comme le rappelle Cosnier par une relation corporelle au monde :

Ainsi c'est par son propre corps que l'on aurait connaissance du corps d'autrui : le corps est non seulement un support essentiel de l'activité mentale, comme le montre son rôle dans l'activité énonciative, mais aussi un instrument essentiel de l'activité relationnelles avec le monde et le autres. L'empathie reposerait fondamentalement sur un substrat corporel. (Cosnier 2006 : 94)

Or les affects exprimés donnent à l'auditeur une perception corporelle de ce type. Le locuteur qui «s'emporte» est senti engagé dans une cause, par le fait que thèse argumentée et pathos ne font qu'un dans le contexte. Le discours politique ne manquera pas d'élaborer des stratégies mettant en place un tel dispositif à travers la charge pathétique dans le but de déclencher une alliance affective envers la cause soutenue. Comme pour n'importe quel effet pragmatique, on ne saurait garantir que l'alliance affective aurait véritablement lieu. Il va toujours de la liberté du destinataire de prendre ses distances et de rejeter la position défendue, qu'il ait perçu ou non la stratégie utilisée et qu'il se soit laissé touché ou non par les affects exprimés. Mais, à tout le moins, les conditions sont mises en place pour qu'opère l'empathie sur la base du transfert métaphorique élaboré plus haut. Il faut d'ailleurs se souvenir que la transmission des affects ne se fait pas uniquement sur un plan individuel, c'est-à-dire de personne à personne, mais concerne également le collectif. Un orateur visera un groupe particulier ou le citoyen en général : cette entité plurielle est appelée à réagir, à partager des affects, bref elle est une cible du pathos tout autant que l'individu. La transmission des affects opère de manière semblable et même s'en trouve favorisée par la circulation d'abondants stéréotypes sur la scène publique. L'écho que produit la réitération de ces configurations figées joue, par le fait de la répétition, le rôle d'insistance dont nous avons fait mention précédemment. Et ceci, sans compter que se dessine une volonté de solidarité avec l'orateur / locuteur qui accompagne l'empathie: une sorte d'automatisme à cimenter l'entité collective, implicite ou explicite, sur laquelle se base les propos publics. 
53 En résumé, le dispositif persuasif à l'œuvre dans les configurations en question tient aux composantes suivantes :

- intensité : choix de procédés associés à la force ;

- empathie, bref une correspondance d'intersubjectivité ;

- projection des particularités du discours sur le contenu pathétique par métaphorisation ;

- performativité : le hic et nunc qui atteste de l'emprise des affects dans le moment du discours.

Voyons deux extraits où une conjonction de procédés pathétiques active cette métaphorisation qui me semble déterminante dans leur rôle persuasif. L'extrait cidessous fait partie d'une chronique parue dans le journal au lendemain de l'élection du gouvernement minoritaire de Pauline Marois au Québec.

(4a) Madame la Première Ministre (j'aime l'écrire au féminin), au-delà de ces processus délibératifs où domine la parole, les citoyens veulent et peuvent agir et assumer des responsabilités. Ils ont des idées. Ils savent créer des alliances pour revitaliser un village. Ils prennent des risques en lançant des entreprises. J'ose vous suggérer de tabler sur cette volonté, de canaliser l'Énergie citoyenne et entrepreneuriale des Québécois, pour transcender les divisions exprimées dans les urnes.

À défaut de pouvoir vous appuyer sur une majorité en Chambre, appuyez-vous sur nous! Faites-nous confiance!

Interpelez vos concitoyens. Invitez-les à se mettre en mouvement. Votre rôle consiste à formuler les objectifs que nous avons en commun puis à créer les conditions de réussite.

(Chronique de Michel Venne, "Appuyez-vous sur nous », Le Devoir, 9 septembre 2012, A7)

Tout en s'appuyant sur l'idée que le citoyen est responsable et que l'on peut faire appel à lui, le chroniqueur y ajoute la force de l'affect. Après avoir énuméré les volontés et capacités des citoyens, il en vient à une recommandation qui prend l'allure de l'insistance, que ce soit sur le mode inclusif ou exclusif. Le pathos fait en sorte que le lecteur-citoyen devrait s'identifier au propos et sentir qu'il fait lui-même cette recommandation à la Première Ministre. La performativité facilite l'empathie.

Un peu plus loin, cette chronique de type épistolaire met en place des procédés semblables et qui génèrent la force. L'asyndète et la répétition de mots et de formes créent un effet d'accumulation, suggérant elle-même l'intensité :

(4b) [...] N'annoncez pas un "plan Marois ». Annoncez un «plan Québec» dans lequel chacun doit mettre l'épaule à la roue, à petite et à grande échelle, et où l'invention et la créativité seront reconnues et appuyées. Appelez-nous à l'action et mettez sur pied les structures d'accompagnement pour que les choses bougent et que l'énergie se canalise en projets constructifs.

(Chronique de Michel Venne, "Appuyez-vous sur nous ", Le Devoir, 9 septembre 2012, A7)

Il me reste à porter un dernier extrait à votre attention.

(5) Nous prenons la parole dans l'espoir de sortir de la torpeur actuelle avant qu'il ne soit trop tard. Un gouvernement seul, de quelque parti que ce soit, ne parviendra pas à vaincre la résistance et l'inertie. Les sonnettes d'alarme doivent retentir dans tous les milieux: politiciens, intellectuels, leaders syndicaux, artistes, gens d'affaires, tous ceux qui aiment le Québec et veulent un avenir prospère pour cet îlot francophone en Amérique doivent prendre la parole. À tous ceux-là, nous lançons un appel à la lucidité, à la responsabilité, à la liberté. 
(Manifeste Pour un Québec lucide, Lucien Bouchard et als, 19 octobre 2005,

pp. 1-10; www.pourunquebeclucide.com)

\section{s'insurge par une proposition d'action et l'appel de ralliement. Contre le silence, il y aurait la parole dénonciatrice et contre l'isolement, il y aurait la solidarité. Ce passage contient tout à la fois l'urgence d'agir et l'insistance auprès de plusieurs instances; ces dernières se trouvent énumérées ("politiciens, intellectuels,...», etc.), faisant jouer l'argument de quantité en faveur du ralliement, ou tout simplement réitérées par le biais de « tous ». Le rythme, l'accumulation d'éléments portés à la thèse défendue scandent le propos tout en servant d'évidence aux affects impliqués (donc à leur transmission) et à la nécessité d'agir. La synergie des procédés en contexte agonistique contribue à l'ampleur. Le champ pathétique ainsi convoqué - et obéissant au dispositif pathétique décrit plus haut - est garant de son efficacité persuasive.}

\section{Conclusion}

Notre maniement du discours, on le sait, est fait d'entorses et de détournements, de dit et de non-dit, d'engagement et d'esquives. Toutes ces postures valent un regard autre que normatif à leur égard, puisqu'elles exploitent d'abord et avant tout les ressources de la langue. Le sens se construit ainsi à partir des éléments codifiés de la langue en ajoutant les traces de la situation énonciative qui nous renseignent sur l'implication du locuteur dans sa prise de parole. Interviennent aussi, à mon sens, le rapport de force entre les interlocuteurs (ou dans la relation orateur / auditoire, auteur / lecteur, etc.), des imaginaires collectifs, des patterns d'organisation textuelle, tout cela avec des charges émotives dont on ne peut faire l'économie. Ils sont à l'œuvre de manière particulière dans ces configurations discursives misant sur le champ pathétique.

Aborder le discours par le biais de sa fonctionnalité argumentative appelle naturellement une extension vers la rhétorique. La visée persuasive se construit sur la planification d'effets à partir d'une mobilisation des moyens psychologiques, cognitifs, idéologiques et autres, ancrés dans la trame linguistique et dans l'usage qui en est fait. La rhétorique a souvent été abordée comme une approche de production, son «art» consistant à suggérer un arsenal de moyens afin de parvenir à l'effet escompté, à savoir l'adhésion du destinataire à la thèse que l'on défend, en d'autres termes, la persuasion. Elle gagne à être pratiquée davantage en tant qu'approche interprétative permettant de dégager le sens impliqué dans un acte énonciatif orienté : étant donné une prise de parole (texte ou discours) à visée persuasive, il s'agit d'établir quel est le rapport immédiat entre les effets et les moyens linguistiques utilisés; quelles sont les incidences de ce rapport sur la construction du sens, celle-ci incluant l'identité, les forces interactionnelles, les positions idéologiques, le renouvellement des imaginaires collectifs, et, bien sûr, la dimension pathétique.

Il va sans dire que mon but n'était pas de produire une analyse exhaustive de tous les procédés linguistiques et rhétoriques inclus dans les quelques extraits cités de cette étude, mais plutôt de montrer le fonctionnement du champ pathétique quand il se met en rapport avec le niveau de l'expression. Du même coup, l'importance de rechercher la valeur argumentative devrait apparaître non seulement, tel que cela se fait souvent, au niveau des entités séparées comme les connecteurs, les lexèmes, mais aussi au niveau transphrastique. Mon étude visait à dégager un certain dispositif persuasif basé sur le 
pathos; bien qu'amené à partir d'un nombre relativement restreint d'extraits par souci de concision, il est évident que ce type de configurations discursives est en usage dans plusieurs autres textes et types de discours. Peu importe la sélection des procédés d'expression et leur agencement, un tel type de configurations à charge pathétique demeure repérable par l'alliance pathos et argumentation mise de l'avant.

Pour revenir au discours politique qui en fait grand usage, on pourra comprendre mieux pourquoi le dispositif proposé, basé sur une alliance du pathos et de l'argumentation, est susceptible de convaincre et même d' " enflammer » les foules: le report des affects sur les propriétés discursives par voie métaphorique est source d'un dynamisme indéniable qui sollicite directement le destinataire, notamment en tirant parti de l'empathie et de la performativité.

\section{BIBLIOGRAPHIE}

Angenot Marc, Dialogue de sourds. Traité de rhétorique antilogique, Paris, Éditions Mille et une nuits, 2008.

Auchlin Antoine, « Compétence discursive et co-occurrence d'affects : ‘blend expérientiels' ou (con)fusion d'émotions? ", in Colletta, Jean-Marc et Anna Tcherkassof (éds), Les émotions. Cognition, langage et développement, Liège, Pierre Mardaga, 2003, pp. 137-152.

Cosnier Jacques, Psychologie des émotions et des sentiments, Paris, Retz-Nathan, 1994. Disponible sur Internet : http://icar.univ-lyon2.fr.

Ducrot Oswald et Anscombre Jean-Claude, L'argumentation dans la langue, Liège, Pierre Mardaga, 1983.

Fontanille Jacques, Sémiotique du discours, Limoges, PULIM, 1998.

Forget, Danielle, Passions bavardes. Essai de rhétorique sur le discours social, Québec, Marcel Broquet éditeur, 2009.

Jakobson Roman, Éléments de linguistique structurale, Paris, Minuit, 1963.

Lakoff George, Women, fire and dangerous things: What Categories reveal about the mind, Chicago, University of Chicago Press, 1987.

Parret Hermann, Essai sur la mise en discours de la subjectivité, Liège, Pierre Mardaga, 1986.

Plantin Christian, «Structures verbales de l'émotion parlée et de la parole émue », in Colletta Jean-Marc et Anna Tcherkassof, Les émotions. Cognition, langage et développement, Liège, Pierre Mardaga, 2003, pp. 97-130.

Raccah Pierre-Yves, «Une sémantique du point de vue : de l'intersubjectivité à l'adhésion », in "L'énonciation identitaire : entre l'individuel et le collectif », dir. par Danielle Forget, Discours social, vol. XXI, 2005, pp. 205-242.

Sénécal Jacques, Manières de dire, manières de penser, Montréal, Liber, 2004, p. 98. 
Simonffy Zsuzsa, « D'un engagement à l'autre ou comment laisser passer la subjectivité d'une instance autre ", in «L'énonciation identitaire : entre l'individuel et le collectif», dir. par Danielle Forget, Discours social, vol. XXI, 2005, pp. 243-259.

Talmy Leonard, Force dynamics in Language and Thought, Parasession on Causative and Agentivity, $21^{\text {th }}$ Regional Meeting, Chicago Linguistic Society, 1985.

Vincent Jean-Didier, Biologie des passions, Paris, Éditions Odile Jacob, 1986.

\section{NOTES}

1. Pour les relations d'empathie voir Auchlin (2003), Cosnier (2006) notamment.

2. Ces configurations sont issues de discours actualisés ; je les considérerai comme des discours écrits : il s'agit, dans la majorité des cas, de retranscriptions et reformulations de discours oraux tenus par des politiciens.

3. Bien sûr qu'elles relèvent aussi du psychique, du psychologique, qu'elles mettent en jeu des questions philosophiques importantes, mais le plan communicationnel joue sur toutes ces facettes et les organise à sa façon. Ce n'est pas tant ce qu'elles sont, comme ce que l'on en fait qui devraient nous inciter à scruter les discours.

4. Sénécal (2004: 98).

5. Voir notamment Parret (1986).

6. Cette relation que l'auteur appelle "corrélation entre intensité et étendue " a pour but de délimiter des qualités sensibles de l'objet et de renommer ainsi les objets en termes de propriétés dynamiques. "Par exemple, c'est au nom de la "solidité" que l'élément terre pourra se voir attribuer une importante force de cohésion et une très faible propension à la dispersion spatiotemporelle ». (Fontanille 1998: 67). Il est difficile de dire si l'on n'est pas en train d'indexer différemment un lexique, de le figer plutôt que de l'ouvrir sur la fluctuation des passions en discours.

7. «Discours » est entendu au sens général de production énonciative en un lieu et temps déterminés.

8. Même si on parle de "mouvements de l'âme »; en effet dans ce cas, c'est l'intériorité qui est visée comme siège du ressenti.

\section{RÉSUMÉS}

Dans cette étude, à l'aide d'un cadre théorique appartenant à l'analyse du discours, j'amorce une réflexion qui se situe à la croisée de la sémantique, de la pragmatique et de la rhétorique, dans cet espace discursif où les énoncés accusent la présence de schémas culturels à forte récurrence. Ces schémas sont en cela reconnaissables lors de la circulation des discours sur la scène publique, en même temps qu'ils reformulent et renouvellent une prise de position idéologique à l'intérieur des nouveaux paramètres textuels qui se présentent. En effet, il faut considérer que ces discours prennent place dans une tension entre le même et le différent qui assure un caractère à la fois consensuel et dynamique à la prise de parole. 
In this study, using a theoretical framework for discourse analysis, I start off a reflection that lies at the intersection of semantics, pragmatics and rhetoric, in this discursive space where utterances present cultural patterns with high recurrence. These patterns are recognizable as the discourse appears in the public realm and at the same time they reformulate and renew an ideological position within the new text parameters. Indeed, one should consider that these discourses take place in a tension between the same and the different which ensure both a consensual and dynamic character to public speaking.

INDEX

Mots-clés : pathos, rhétorique, passion, argumentation, affects, dispositif persuasif

Keywords : pathos, rhetoric, passion, argument, affects, persuasive apparatus

\section{AUTEUR}

DANIELLE FORGET

Université d'ottawa 